\title{
Euclidean space and their functional application for computation analysis
}

\author{
Jay Prakash Tiwari" and Manish Pande \\ Professor, Patel College of Science \& Technology, Indore, Madhya Pradesh
}

Received: 11-April-2019; Revised: 16-July-2019; Accepted: 20-July-2019

(C2019 Jay Prakash Tiwari and Manish Pande. This is an open access article distributed under the Creative Commons Attribution (CC BY) License, which permits unrestricted use, distribution, and reproduction in any medium, provided the original work is properly cited.

\begin{abstract}
Euclidean space is the calculative procedure for the calculation of the inner and outer points. The set of points for the calculation is called the Euclidean space. It is also used in the form of Euclidean distance. It is used in different domains and in their applications. It is widely used for the clustering and classification algorithms along with the wide applicability in statistics. In the prospect of the above view the Euclidean space and their functional application have been explored and discussed in this paper. The main aim is to focus and highlights the area of applicability along with the study of the computational feasibility by discussing the previous work.
\end{abstract}

\section{Keywords}

Euclidean space, Distance points, Functional application, Computation analysis.

\section{Introduction}

Euclidean space is the collection of tuples or the points which can be used for the exploration of the boundaries in the data statistics for different purpose and in different domains. It has been widely used with the communication system [1-3]. The communication between the nodes and the applicability with the shortest route has emphasized the research development in this field [4-8].

There are several applications including the image recognition, gene expression profiles, design and modeling, antenna and communication systems [9-11]. It is also wide applicability in different engineering applications [12].

Dubey et al. also used the distance space in different ways and proved the applicability of the improved accuracy and better detection framework [13-14]. They have used these distance spaces with the clustering methods. The applicability can be extended in terms of the transmission system and the initial block submission for granting the source and receiver the same data points or the nodes. It is also used for the autocorrelation properties [15]. Figure 1 shows the Euclidean space-based method linkage. Figure 2 shows the Euclidean space-based data hierarchy.

\footnotetext{
*Author for correspondence
}

The main objectives of this paper are as follows:

1. To explore and analyze the areas of usability for the application development and the improvement in the results.

2. Focus on related research work in the same domain based on computational aspects.

3. To discuss the fundamental aspects with the advantages and provides the solutions for the gaps.

\section{Literature review}

In 2007, Sharma and Papadias [16] discussed the rotated constellations in terms of the literature in the area of quasi-orthogonal space-time codes. They have suggested it is important to ensure the full diversity. They have suggested the need of optimization of the rotation to get the exact probability of error. The product criteria are based on the probability of error. They have provided an analytical view for the optimal rotation angles.

In 2011, Shao et al. [17] discussed the application of the shortest raster distance algorithm. It is discussed on the basis of the Euclidean space. Their aim is to obtain the shortest Euclidean distance. Their approach is based on multiple point sources and polygonal obstacles based. First the turning focuses on the courses are characterized as the unrelated focuses on the digression lines from guide sources toward obstructions raised. At that point, search the raster cells as per certain standards of defining 
moments, what's more, interface them to fabricate the courses which present the briefest separations from the sources to the goals bypassing obstructions. The key procedures of this calculation are to identify whether a straight course is crossing an obstruction, to characterize defining moments, what's more, to make guesses to the goal through turning focuses. The outcomes demonstrate that the calculation is steady and precise.

In 2013, Songhao et al. [18] presented a high order statistic based maximum a posterior (HOS-MAP) approach. This approach provides the dissimilarity representations in a modified pseudo-Euclidean space. At that point, the data of the difference increases appropriation of every classification is accomplished depends on high-request insights of triplets of neighbor focuses for each picture information. At last, a greatest a posteriori calculation with the data of Gaussian Mixture Model and tripletdivergence increases dispersion is embraced to evaluate the pertinence of every classification in the database for each information new picture. Test results on a broadly useful picture database show that viability and effectiveness of the proposed MAPHOR conspire.

In 2015, Ma and An [19] discussed the long Euclidean geometry LDPC codes. The basis of the discussion is the space missions. They have analyzed the encoding algorithm first. They have considered incidence vector of points and lines for the paritycheck matrix in Euclidean geometry. For the generator matrix computation, it is derived from solving matrix equation over GF (2). They have proposed a new encoding circuit of using feedback shift registers based on the quasi-cyclic matrix. They have designed lLast, serial encoding circuit for resource-optimization and parallel encoding circuit for speed optimization. It is performed on Xilinx XC4V SX55 FPGA. Their results indicate that the encoding scheme is efficient for the space communications.

In 2015, Gong et al. [20] discussed the problem of routing algorithm on hexagonal networks. They have created an oblique coordinate system for addressing the hexagonal nodes based on Euclidean space. Then they have developed optimal routing algorithms. It is developed by using the vectors and angles of the redefined Euclidean space. They have compared their approach with the traditional 3-directions scheme and the Cayley graph method. Their results indicate that their algorithm is more efficient. It is also totally independent. They have also proved that the path is short in all the complete experimentation.

In 2015, Nasrullah and Varma [21] discussed about the routing. They have suggested large overhead due to routing. Due to this overhead the performance and the scalability of the network degrades. They have suggested that the geographic routing protocols are highly scalable because of the stateless routing. They have discussed the inconsistency between the two topologies. According to the authors the performance of the network increases if the networks are embedded in Hyperbolic Space rather than Euclidean Space. They have evaluated the performance of greedy routing. The main basis of comparison is hyperbolic space and Euclidean space. Their evaluation is based on success percentage and hop stretch metrics.

In 2016, Lavrovska and Rassomahin [22] considered and emphasized on the main problem of pseudorandom codes use. It has been considered in terms of systems responsible for the data transmissions. They have developed the pseudo-random codes in Euclidean space. Their model shows like bearers of code words as bits of acknowledgment of pseudorandom forms with a restricted range.

In 2018, Afrasiyabi et al. [23] presented an approach based on the non-Euclidean vector product. It is applied for the artificial neural networks. They have proposed a class of neural networks. It is proposed with the universal approximation property. The Lebesgue integrable functions have been used. It is based on the non-Euclidean vector product. Their results support the proposed method betterment in comparison to the comparable methods.

In 2018, Luchin et al. [24] discussed the problems of co-located radio direction finding. The discussion is based on the polarization-selective reception. They have explored the direction finder and the directionfinding algorithm. It is based on the four-element antenna system. The four-component receiving wire has a tri-symmetrical circle receiving wire joined with a vertical dipole, and permits four symmetrical directional examples to be shaped with a solitary stage focus. It has discovered that utilizing of proposed radio wire framework makes it conceivable to play out all sign handling which is fundamental for course finding in the four-dimensional Euclidean space of sign vectors (expanded sign space) in examination with the known co-found bearing discoverers in light of three-symmetrical receiving 
wires. The hypothetical examination with evidence of primary theories, which are hypothetical establishment of the bearing discovering calculation in states of vulnerability and transitory precariousness of got signal polarization circles have been considered. Their results show that the increase of the signal space produces positive effect.

In 2018, Qiao et al. [25] discussed about the action recognition system. They have suggested that the corresponding space structures of video representation are ignore in the previous research work. They have addressed the action recognition task with a spatial-temporal representation analysis algorithm with Grassmannian manifold and Euclidean space (ST-AGE). Temporal features have been extracted from each video sample. It has been done with the trajectory tracking method. It is analyzed in the context by embedding them. They have conducted the Grassmannian manifold and
Euclidean space with the multi-kernel SVM. The results support the approach.

In 2018, Zhang et al. [26] discussed the mismatch between the training (source) and testing (target) datasets by this the performance effects of any model. They have suggested that most of the algorithms reduce the distribution discrepancy in the input spaces for this problem. They have suggested the statistical properties are important in case of kernelbased learning machine that is reproducing kernel Hilbert spaces (RKHS). They have proposed a novel strategy for matching distributions in RKHS. It is achieved by RKHS covariance alignment. This strategy is a based on the Euclidean spaces to infinite-dimensional feature spaces. They have provided two alignment approaches. By this they have achieved closed-form expressions via kernel matrices. Their results show that their approaches outperform in both accuracy and efficiency.

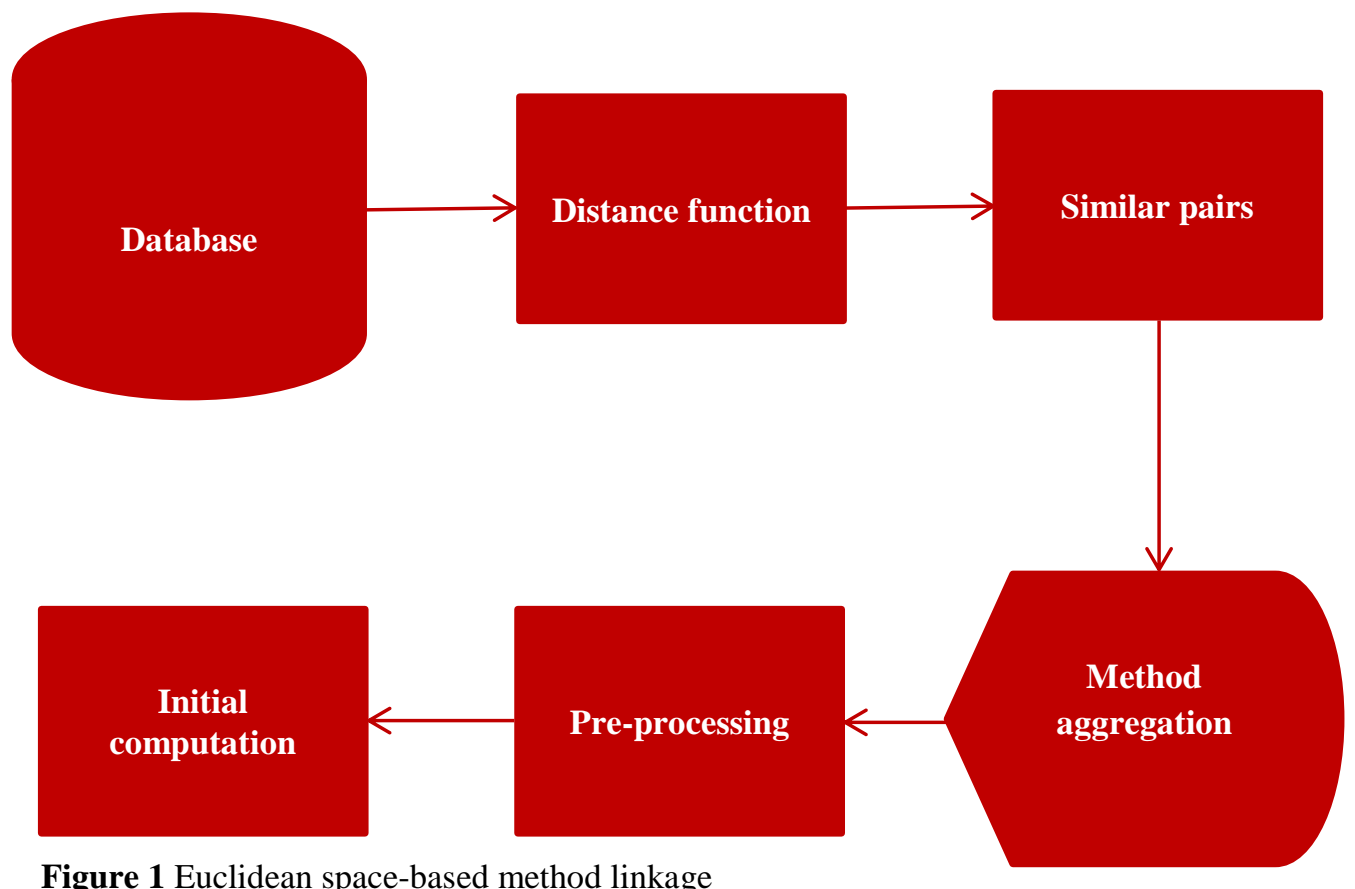

Figure 1 Euclidean space-based method linkage 


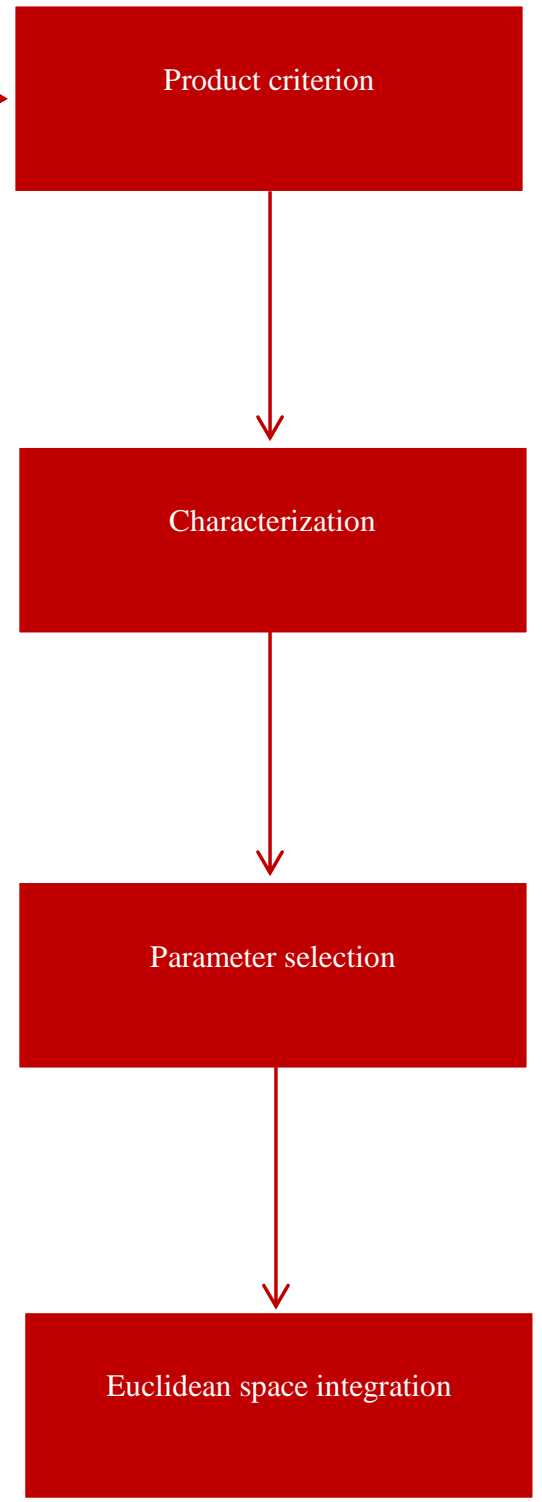

Figure 2 Euclidean space-based data hierarchy

\section{Problem domain}

Based on the discussion and analysis of the previous research works, the following statements have been identified.

1) There is the need of data mining linkage with the Euclidean space for the better pattern point discovery in several domains.

2) There is the need of working on autocorrelation.

3) There is the need of error estimation in terms of data points and space disjoints.

4) There is the need of the considerations of the region of curve, regression and coefficient

vectors in terms of attribute and parameter variations.

5) There is the need of optimization linkup with the Euclidean space for the complex problem.

\section{Analysis}

In this paper different literature related to the Euclidean space with different application areas has been discussed and analyzed. The main parameters are method and the results evaluation parameters. The comparison of the latest methods highlighted the impacts have been discussed in Table 1. 
Jay Prakash Tiwari and Manish Pande

Table 1 Comparative analysis

\begin{tabular}{llll}
\hline S.No & Reference & Method & Computation approach \\
\hline 1 & Xu and Liu [27] & $\begin{array}{l}\text { Spacelike translating } \\
\text { solitons }\end{array}$ & $\begin{array}{l}\text { They have investigated the parametric version and non-parametric version } \\
\text { of rigidity theorem of spacelike translating solitons in pseudo-Euclidean } \\
\text { space. }\end{array}$ \\
\hline 2 & Lima et al. [28] & $\begin{array}{l}\text { Hypersurfaces } \\
\text { in Euclidean Space }\end{array}$ & $\begin{array}{l}\text { They have provided a classification. It is of the generalized translation } \\
\text { graphs with constant mean curvature. This is also called Gauss-Kronecker } \\
\text { curvature. }\end{array}$ \\
\hline 3 & Fampa [29] & $\begin{array}{l}\text { Steiner minimal trees in } \\
\text { Euclidean space }\end{array}$ & $\begin{array}{l}\text { They have presented properties related to the topology of Steiner minimal } \\
\text { trees. They have also investigated the application and the geometric } \\
\text { position of Steiner points. }\end{array}$ \\
\hline 5 & $\begin{array}{l}\text { BenKhlifa and } \\
\text { Ghorbel [30] }\end{array}$ & $\begin{array}{l}\text { Complete curvature } \\
\text { scale } \\
\text { representation }\end{array}$ & $\begin{array}{l}\text { They have proposed local shape curve features. It is useful and invariant } \\
\text { under planar Euclidean transformations. It is also independent with } \\
\text { respect to the curve parameterization. }\end{array}$ \\
\hline 5 & Han [31] & $\begin{array}{l}\text { MA-digitization of 2D } \\
\text { Euclidean spaces }\end{array}$ & $\begin{array}{l}\text { They have presented a homotopy. They named the homotopy as LMA- } \\
\text { homotopy and provides the investigation based on the LMA-homotopy } \\
\text { equivalence for the Euclidean topology and M-topology. }\end{array}$ \\
\hline 6 & $\begin{array}{l}\text { Deshmukh and } \\
\text { Al-Dayel [32] }\end{array}$ & $\begin{array}{l}\text { Immersion in Euclidean } \\
\text { spaces }\end{array}$ & $\begin{array}{l}\text { They have studied and discussed the compact immersed orientable } \\
\text { hypersurfaces in the Euclidean space. They have discussed the suitability } \\
\text { based on the tangential and normal components. }\end{array}$ \\
\hline
\end{tabular}

\section{Conclusion and future suggestions}

In this paper four-way aspects have been used for the study and discussion. In the first way the research paper has been identified from different domains related to Euclidean space. In the second way the methods have been indented for the discussion. In the third aspects the parameters and the attributes used have been discussed with the advantages and disadvantages. Finally, based on the study solutions and impact have been discussed, analyzed and deep explorative computation link up has been considered for the combination.

The future work can be categorized as follows:

1. The impact of the combination with the different methodology in communication may be analyzed and discovered with the coding and encoding mechanism.

2. It can be combined to find the usability with the computational algorithms like clustering and classification.

3. The impact of the combination of these methods' applicability may be future research work.

\section{Acknowledgment}

None.

\section{Conflicts of interest}

The authors have no conflicts of interest to declare.

\section{References}

[1] Singh H, Urrutia J. Compass routing on geometric networks. In proceedings of the Canadian conference on computational geometry 1999 (pp. 51-4).

[2] Huang H, Yin H, Luo Y, Zhang X, Min G, Fan Q. Three-dimensional geographic routing in wireless mobile ad hoc and sensor networks. IEEE Network. 2016; 30(2):82-90.

[3] Kuhn F, Wattenhofer R, Zollinger A. Worst-case optimal and average-case efficient geometric ad-hoc routing. In proceedings of the international symposium on mobile ad hoc networking \& computing 2003 (pp. 267-78). ACM.

[4] Bose P, Morin P, Stojmenović I, Urrutia J. Routing with guaranteed delivery in ad hoc wireless networks. Wireless Networks. 2001; 7(6):609-16.

[5] Cvetkovski A, Crovella M. Hyperbolic embedding and routing for dynamic graphs. In INFOCOM 2009 (pp. 1647-55). IEEE.

[6] Kou Y, Lin S, Fossorier MP. Low-density paritycheck codes based on finite geometries: a rediscovery and new results. IEEE Transactions on Information Theory. 2001; 47(7):2711-36.

[7] Tanner RM. Spectral graphs for quasi-cyclic LDPC codes. In proceedings of the international symposium on information theory 2001 (p. 226). IEEE.

[8] Luchin DV, Trofimov AP, Filippov DV, Yudin VV. Algorithm of radio direction finding in the HF range in polarization fading conditions with collocated antennas. In Moscow workshop on electronic and networking technologies 2018 (pp. 1-8). IEEE.

[9] Chaudhari V, Patil CY. Disease detection of cotton leaves using advanced image processing. International Journal of Advanced Computer Research. 2014; 4(15):653-9.

[10] Sikandar M. Design and modeling of a 3 DOF machine. International Journal of Advanced Computer Research. 2014; 4(15):611-7.

[11] Marie F, Erhel Y, Bertel L, Lemur D. Design of a HF compact direction-finding system based on colocated antennas. In eighth international conference on $\mathrm{HF}$ radio systems and techniques 2000 (pp. 127-31). IET.

[12] Tiwari JP, Pande M. Boolean algebra and harmonic function-based computation analysis: a survey and analysis. International Journal of Advanced 
Technology and Engineering Exploration. 2019; 6(53):107-11.

[13] Dubey AK, Gupta U, Jain S. Analysis of k-means clustering approach on the breast cancer Wisconsin dataset. International Journal of Computer Assisted Radiology and Surgery. 2016; 11(11):2033-47.

[14] Dubey AK, Gupta U, Jain S. Comparative study of kmeans and fuzzy c-means algorithms on the breast cancer data. International Journal on Advanced Science, Engineering and Information Technology. 2018; 8(1):18-29.

[15] Stasev YV, Kuznetsov AA, Nosik AM. Formation of pseudorandom sequences with improved autocorrelation properties. Cybernetics and Systems Analysis. 2007; 43(1):1-11.

[16] Sharma N, Papadias CB. Euclidean distance maximizing rotations for quasi-orthogonal space-time codes with MPSK symbols. In international symposium on personal, indoor and mobile radio communications 2007 (pp. 1-5). IEEE.

[17] Shao Z, Ma Q, Liu X, Ma J. An algorithm for shortest raster distance in Euclidean space with obstacles. In international conference on geoinformatics 2011 (pp. 1-4). IEEE.

[18] Songhao Z, Juanjuan H, Wei S. Image classification using three order statistics in non-Euclidean spaces. In Chinese control and decision conference 2013 (pp. 6973). IEEE.

[19] Ma M, An J. Algorithms and implementation of long Euclidean-geometry LDPC codes for space communications. In international conference on information science and control engineering 2015 (pp. 100-4). IEEE.

[20] Gong J, Li L, Chen G, Wu Y, Li J. An addressing and routing scheme based on modified Euclidean space for hexagonal networks. China Communications. 2015; 12(5):94-9.

[21] Nasrullah S, Varma S. Greedy routing performance analysis of network embedded in hyperbolic and Euclidean Space. In international conference on computing and network communications 2015 (pp. 491-5). IEEE.

[22] Lavrovska T, Rassomahin S. Physical model of pseudorandom codes in multidimensional Euclidean space. In international scientific-practical conference in communications science and technology 2016 (pp. 67-70). IEEE.

[23] Afrasiyabi A, Badawi D, Nasir B, Yildi O, Vural FT, Çetin AE. Non-Euclidean vector product for neural networks. In international conference on acoustics, speech and signal processing (pp. 6862-6). IEEE.

[24] Luchin DV, Trofimov AP, Yudin VV, Spodobaev MY, Filippov DV. Co-located radio direction finding in polarization fading conditions with increased Euclidean space dimension of signal vectors. In systems of signal synchronization, generating and processing in telecommunications 2018 (pp. 1-5). IEEE.

[25] Qiao X, Zhou C, Xu C, Cui Z, Yang J. Action recognition with spatial-temporal representation analysis across Grassmannian manifold and Euclidean space. In international conference on image processing 2018 (pp. 3448-52). IEEE.

[26] Zhang Z, Wang M, Huang Y, Nehorai A. Aligning infinite-dimensional covariance matrices in reproducing kernel Hilbert spaces for domain adaptation. In proceedings of the conference on computer vision and pattern recognition 2018 (pp. 3437-45). IEEE.

[27] Xu R, Liu T. Rigidity of complete spacelike translating solitons in pseudo-Euclidean space. Journal of Mathematical Analysis and Applications. 2019; 477(1):692-707.

[28] Lima BP, Santos NL, Sousa PA. Generalized translation hypersurfaces in Euclidean space. Journal of Mathematical Analysis and Applications. 2019; 470(2):1129-35

[29] Fampa M. Insight into the computation of Steiner minimal trees in Euclidean space of general dimension. Discrete Applied Mathematics. 2019.

[30] Benkhlifa A, Ghorbel F. An almost complete curvature scale space representation: Euclidean case. Signal Processing: Image Communication. 2019; 75:32-43.

[31] Han SE. Homotopic properties of an MA-digitization of 2D Euclidean spaces. Journal of Computer and System Sciences. 2018; 95:165-76.

[32] Deshmukh S, Al-Dayel I. Characterizing spheres by an immersion in Euclidean spaces. Arab Journal of Mathematical Sciences. 2017; 23(1):85-93.

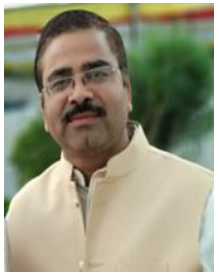

Electrical.

Email: mrjaytiwari31@gmail.com

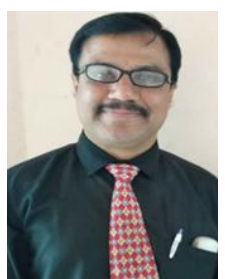

Dr. Manish Pande completed his Ph.D from Government MGCGV, Chitrakoot University Chitrakoot, MP under the faculty of Science. He is currently working as a Professor in the Patel Group of Institutions, Indore, MP. His research interests are all related topic of Mathematics in the area of Computer, Electronics and Electrical. 